\title{
Analysis of the Possibilities for Using a Uniform Bragg Grating in a Tunable Dispersion Compensator
}

\author{
Waldemar Wójcik, Piotr Kisała, Gulbahar Yussupova, Nazym Kussambayeva, \\ Gulzhan Kashaganova and Damian Harasim
}

\begin{abstract}
The article presents a tunable fibre optic dispersion compensator system, consisting of a specially designed cantilever beam and a uniform Bragg grating. It analyses the group delay and dispersion characteristics in the case that there is no apodization of the grating and also for a grating with apodization used for modulation of the refractive index. Various apodization parameters were tested, along with their effects on the dispersion characteristics of the entire system properties. It is demonstrated in the paper that the apodization parameter affects the compensator's group delay characteristic. The finite elements method was used to design a compensator of such a shape that enabled chirp to be induced in a grating of a specified shape. A new design is presented for the system, in which the dispersion properties are tuned by the maximum value of the heterogeneous deformation of the compensator. The paper also includes results showing the effect of the maximum value of heterogeneous stress of the grating on the dispersion characteristics of the proposed construction.
\end{abstract}

Keywords - dispersion compensation, group delay, fiber Bragg gratings, tunable dispersion compensator

\section{INTRODUCTION}

$\mathbf{C}$ OMMUNICATION by means of an optic medium has many advantages, but the main factor limiting its efficiency is dispersion. There are many methods of dispersion compensation. The dispersion compensation fibres in use have a flaw in the form of the compensator's large size [1]. Another disadvantage is the constant length of compensating fibre required for compensation ina fibre optic connection of a specified length, which means that these solutions cannot be applied in networks with optical switching, where the length of the optical connection may vary. Coupled two-cavity all-pass filters realised entirely as thin-film structures for dispersion slope compensation of optical fibres have been proposed [2]. Liquid crystals used in optical sensors $[3,4]$ have also been proposed for the polarization mode dispersion compensation in the Liquid-Crystal Modulator Arrays [5]. Among systems currently used, those based on fibre Bragg gratings are also

W. Wójcik, P. Kisała D. Harasim are with the Lublin University of Technology, Institute of Electronics and Information Technology, Lublin, Poland (email: waldemar.wojcik@pollub.pl, p.kisala@pollub.pl, d.harasim@pollub.pl)

G. Yussupova ${ }^{2}$ N. Kussambayeva ${ }^{2}$ G. Kashaganova are with the Kazakh National Research Technical University after K. I. Satpaev, Almaty, Kazakhstan (email: .gulbahar68@mail.ru; kussambayeva@mail.ru; guljan_k70@mail.ru). worth mentioning [6-8]. The basic flaw of such systems is the fact that the grating of the compensator only works for one wavelength.

A solution may be to tune the grating in order to adapt its compensatory properties to a specific wavelength for other requirements, e.g. to obtain a defined scope of group delay [9,10]. Chirped [11], non-linear chirped [12], apodized [13] or sampled [14,15] Bragg gratings are most commonly used in the case of dispersion compensators. The influence of the grating parameters on its third-order dispersion value have also been studied [16]. In some cases the acoustic effect allows control of the dispersion using a uniform FBG [17].

The present article presents proposals to use a uniform Bragg grating in the construction of a tunable fibre optic dispersion compensator. The influence of the apodization profile of a homogeneous Bragg grating on the group delay of the signal in the area of the grating was measured. A special mounting has been proposed for the grating on a specially prepared cantilever beam allowing chirping to be induced in the grating by force being applied. The analysed response to the designed compensator enabled the grating apodization parameter to be identified which allows the optimum spectral characteristics and dispersal properties to be obtained. The first laboratory experiments and simulation tests were conducted using coupled mode theory, and the effect of the parameter of Gaussian distribution of the grating's apodization function on its dispersion characteristics. The optical qualities of the system analysed are given as graphs of the group delay and the reflection and transmission spectrum of the gratings in the wavelength function. Because grating's strain and temperature variations cause a change to Bragg's wavelength, in future works the temperature compensations methods should be applied [18-20].

\section{MODEL OF A DISPERSION COMPENSATOR}

In the case of a homogeneous grating, the modulation of the refractive index is approximately homogeneous in the entire grating located within the fibre core, and there are no propagation modes outside the core. With this in mind, plane mode scan be disregarded. The distribution of the electric field along the fibre core can thus be expressed as follows:

$$
E(x, y, z)=[A(z) \exp (-i \beta z)+B(z) \exp (-\beta z)] e_{t}(x, y)
$$


where $A(z)$ and $B(z)$ are the amplitudes of the propagation modes in directions $+\mathrm{z}$ and $-\mathrm{z}$ respectively. The value $z$ denotes the axis along which the Bragg grating is written, $\beta$ is the propagation constant. Introducing the distribution $E(x, y, z)$ to the coupled mode equations [21] results in the following:

$$
\begin{gathered}
d R(z) / d z=i \sigma(z) R(z)+i \kappa(z) S(z), \\
d S(z) / d z=-i \sigma(z) S(z)+i \kappa *(z) R(z),
\end{gathered}
$$

where:

$$
\begin{gathered}
R(z)=A(z) \exp [i(\delta z-\varphi / 2)], \\
S(z)=B(z) \exp [-i(\delta z+\varphi / 2)] .
\end{gathered}
$$

Here $R(z)$ represents the mode moving in direction $+z$, and $S(z)$ the mode moving in the opposite direction, i.e. $-z, \quad \sigma$ is the direct component (DC) of the modulation of the refractive index, $\kappa(z)$ is the alternate component (AC) of the modulation of the refractive index in the grating (also known as local grating strength). Equations (2) and (3) were used to construct the model of the Bragg dispersion compensator in order to obtain the dispersion and group delay characteristics in the grating. These parameters are also paramount when calculating the parameters of the proposed system.

In the paper, the refractive index of the light in the grating is assumed as a value dependent on $z$, we can thus say that:

$$
n(x, y, z)=n(z)=n_{0}+\delta n_{0}+\delta n(z) \cos (2 \pi z / \Lambda+\varphi(z))
$$

where $n_{0}$ is the refractive index of the light in the core on the section of fibre without the grating, $\delta n_{0}$ is the depth of modulation of the refractive index, $\delta n(z)$ is the amplitude of the modulation of refractive index, $\Lambda$ is the grating period, $\varphi$ is the grating chirp (meaning the dependence of the phase of the grating on the position along axis $\mathrm{z}$ ). The values from the dependencies described by equation (6) are also presented in figure 1 .

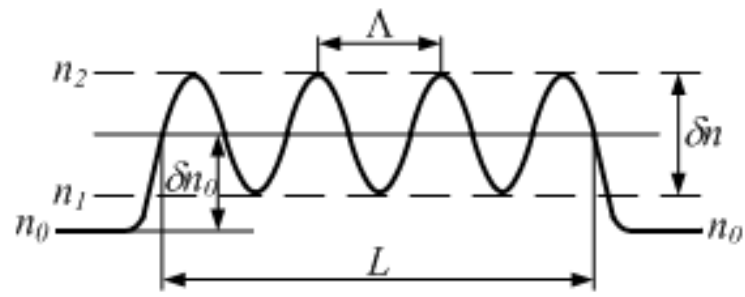

Figure 1. Designation of the characteristic parameters of the modulation of refractive index in the model dispersion compensator

The value $n_{l}$ represents the minimal value of the grating's refractive index, while $n_{2}$ denotes the maximum value of the grating's refractive index and $L$ denotes its length. The paper assumes a grating period described as a third degree polynomial:

$$
\Lambda=\Lambda_{0}\left(b_{1} \cdot z^{3}+b_{2} \cdot z^{2}+b_{3} \cdot z+b_{4}\right) .
$$

Figure 2 presents the distribution of the changes of the period along the length of the grating.

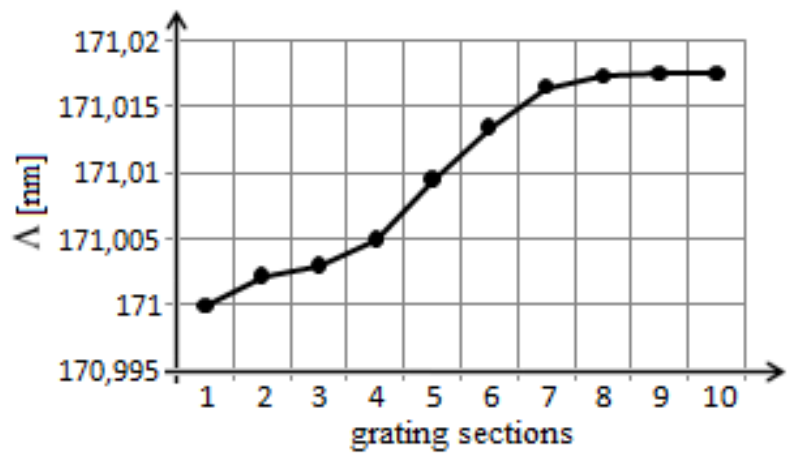

Figure 2. Change in the period length of the grating used in the dispersion compensator

For the purposes of solving the coupled mode equations the self-coupling coefficient ois assumed as equal to:

$$
\sigma=\delta+\overline{\delta n_{\text {eff }}} 2 \pi / \lambda-0.5 d \phi / d z
$$

where $\delta$ is the parameter defining the amount of detuning, $\overline{\delta n_{e f f}}$ is the ,dc" index change and is averaged over the extent of each period of the grating, while $d \phi / d z$ defines the grating chirp, connected with the change induced in its periods, according to fig. 2 . The detuning parameter was defined based on the following relationship:

$$
\delta=\beta-\pi / \lambda=\beta-\beta_{B}=2 \pi n_{e f f}\left(1 / \lambda-1 / \lambda_{B}\right),
$$

where $\beta$ denotes the propagation constant, while the Bragg wavelength is expressed as the relationship:

$$
\lambda_{B}=2 n_{e f f} \Lambda
$$

where $n_{\text {eff }}$ is the effective refractive index in the fibre core. The calculations also assume that the coupling coefficient $\kappa(z)$ can be represented by:

$$
\kappa=(\pi / \lambda) \delta n(z) g(z) v,
$$

where $g(z)$ is the function of the apodization, and $v$ is fringe visibility.

The grating was divided into ten sections, each of which was represented by means of a separate state-transition matrix. The process of propagating light through the entire grating is described by equation (12).

$$
\left[\begin{array}{c}
A_{\text {in }} \\
B_{\text {out }}
\end{array}\right]=\left[\begin{array}{cc}
\frac{1}{t} & \frac{r^{*}}{t^{*}} \\
\frac{r}{t} & \frac{1}{t^{*}}
\end{array}\right]\left[\begin{array}{c}
A_{\text {out }} \\
B_{\text {in }}
\end{array}\right],
$$


where $r=|r| \exp \left(i \phi_{r}\right)$ and $t=|t| \exp \left(i \phi_{t}\right)$ correspond to the reflection and transmission coefficients, $\phi_{r}$ and $\phi_{t}$ are the phases of the FBG's reflection and transmission coefficients. The denotations from equation (12) are presented in figure 3 :

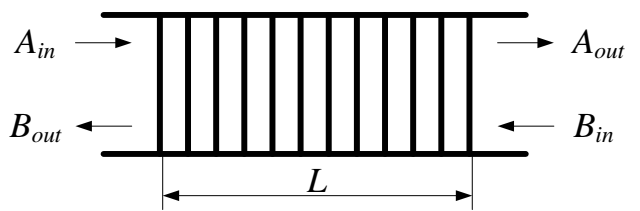

Figure 3. Denotations of signals in the dispersion compensator

Thus the whole state-transition matrix, taking into consideration the matrices of the individual sections, looks like this:

$$
\left[\begin{array}{c}
A_{\text {in }}{ }^{i} \\
B_{\text {out }}{ }^{i}
\end{array}\right]=T^{i} \times\left[\begin{array}{c}
A_{\text {out }}{ }^{i-1} \\
B_{\text {in }}{ }^{i-1}
\end{array}\right]=\left[\begin{array}{cc}
T_{11}{ }^{i} & T_{12}{ }^{i} \\
T_{21}{ }^{i} & T_{22}{ }^{i}
\end{array}\right] \times\left[\begin{array}{c}
A_{\text {out }}{ }^{i-1} \\
B_{\text {in }}{ }^{i-1}
\end{array}\right],
$$

where index $i$ denotes the number of the grating section, while the individual matrices are expressed as follows:

$$
\begin{gathered}
T_{11}{ }^{i}=\cosh \left(\gamma^{i}\right)-i(\sigma / \gamma) \sinh \left(\gamma z^{i}\right), \\
T_{21}{ }^{i}=i(\kappa / \gamma) \sinh \left(\gamma z^{i}\right), \\
T_{22}{ }^{i}=T^{*}{ }^{*}{ }^{i}, \\
T_{12}{ }^{i}=T^{*}{ }_{21}{ }^{i},
\end{gathered}
$$

while

$$
\left\{\begin{array}{ll}
\gamma=\sqrt{\kappa^{2}-\sigma^{2}} & \kappa^{2}>\sigma^{2} \\
\gamma=i \sqrt{\sigma^{2}-\kappa^{2}} & \kappa^{2}<\sigma^{2} .
\end{array},\right.
$$

Taking into account the fringe conditions, i.e. $A_{\text {in }}=1$ and $B_{i n}=0$, from equation (19) the grating's responses were denoted in the form of the transmission spectrum $A_{\text {out }}$ and reflection spectrum $B_{\text {out }}$ :

$$
\left[\begin{array}{c}
A_{\text {in }} \\
B_{\text {out }}
\end{array}\right]=T_{N} \cdot T_{N-1} \cdot \ldots \cdot T_{i} \cdot \ldots \cdot T_{1} \times\left[\begin{array}{c}
A_{\text {out }} \\
B_{\text {in }}
\end{array}\right],
$$

The paper analyses the work of a dispersion compensator with a non-apodized uniform grating, and with a grating apodized with a Gauss profile, with the entire profile being described by the relationship:

$$
g(z)=\exp \left[-a((z-L / 2) / L)^{2}\right]
$$

where $L \mathrm{~s}$ the grating length, $z \in[0, L], a$ is the Gauss parameter.

\section{METHOD OF CHIRP GENERATION IN THE DISPERSION COMPENSATOR}

In order to generate a linearly variable period for the Bragg grating which causes chirp to occur in the grating, the grating was stuck onto a specially designed cantilever beam. This cantilever beam was then subjected to tensile stress which resulted in deformations appearing in the grating. The shape of the cantilever beam and the point where the dispersion compensator grating was attached are shown in figure 4. As can be seen in figure 5 , one end (marked B) was placed immobile, while the second(marked A) was subjected to the influence of force which caused it to become deformed. The nature of the deformations in the fibre optic fibre on which the Bragg grating was written is illustrated in figure 5.

The deformation values were calculated using the finite elements method. As can be seen, the character of the deformation curve corresponds to the polynomial described by equation(7). In the case presented in figure 6 , the deformation curve is described by the equation:

$$
\varepsilon=c_{1} \cdot z^{3}+c_{2} \cdot z^{2}+c_{3} \cdot z+b_{4},
$$

where the values of the individual constants are $c_{1}=0.002, c_{2}=0.0293, c_{3}=-0.0232, c_{4}=1.252$.

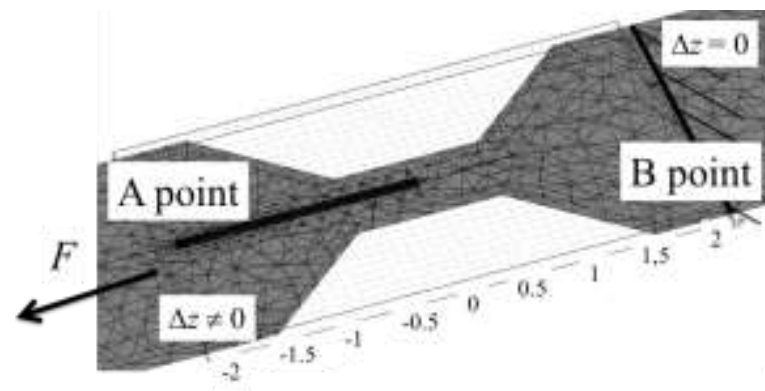

Figure 4. Grating of finished elements, applied to the dispersion compensator system consisting of a cantilever beam and Bragg grating. End A: subjected to tensile stress F, End B: firmly attached, immovable

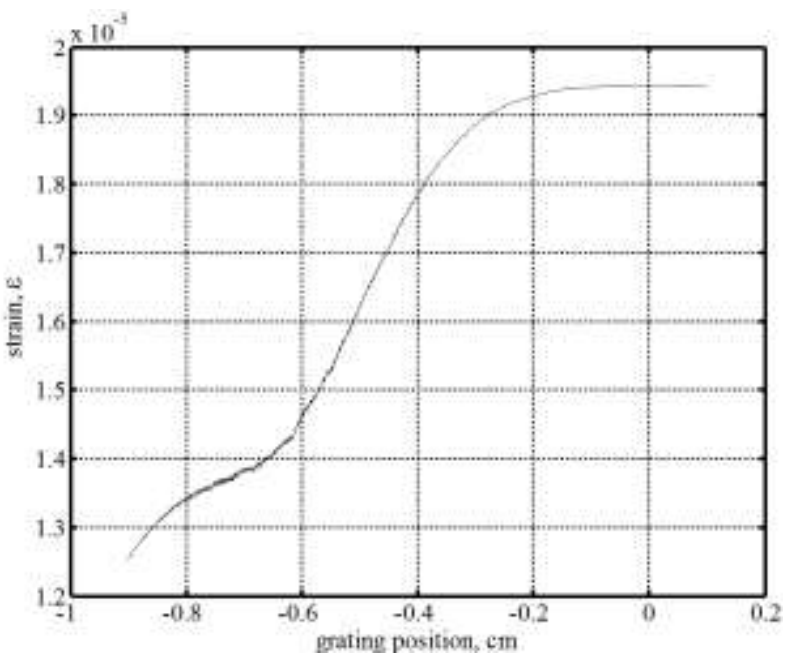

Figure 5. Distribution of deformation values along the grating. $0.9-$ beginning of grating, +0.1 - end of grating 


\section{RESULTS}

In order to establish the dispersion properties of the proposed system, the group delay and dispersion values were calculated. The calculations were carried out assuming the real values of the parameters of the homogeneous grating used as a compensator. A grating with a length of $L=5 \mathrm{~cm}$ and effective refractive index of $n_{\text {eff }}=1.447$ was used. The Bragg grating was mounted on an element which induced chirp in it with constant force. Its Bragg wavelength was $\lambda_{B}=1554.25 \mathrm{~nm}$. Figure 6 shows the grating's transmission and reflection spectrum, while there is a graph of the group delay in figure 7 .

Figure 8 presents the dispersion characteristic where there is no apodization or force causing a heterogeneous period to be generated on the length of the grating. Analogous graphs of the transmission spectrum and of group delay and dispersion in the event that the grating is apodized are given in figures 9-11.

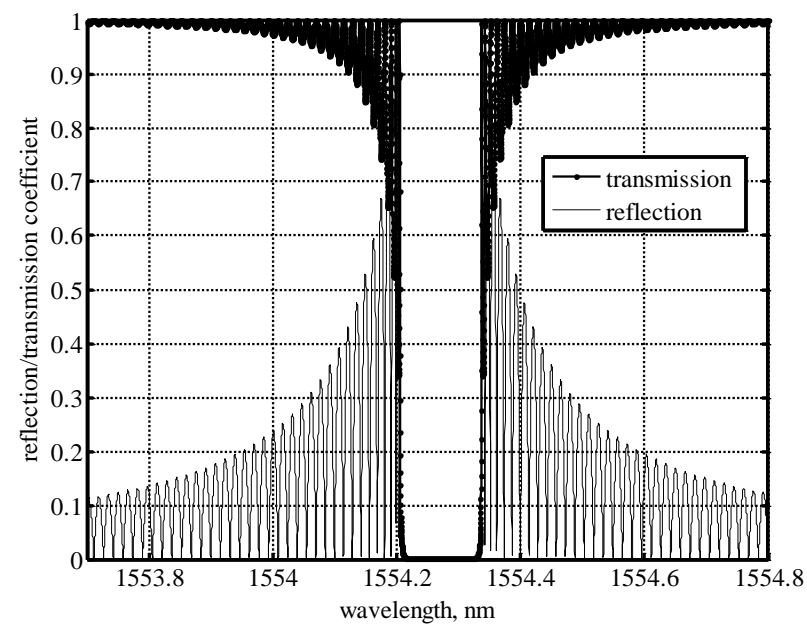

Figure 6. Spectral characteristics of transmission and reflection in the case of a non-apodized grating, no grating chirp is caused

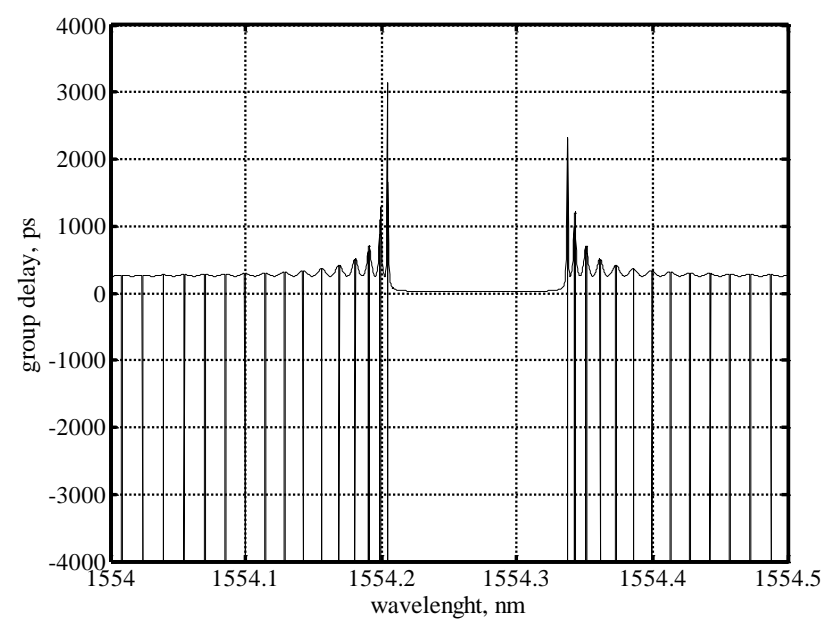

Figure 7. Group delay where there is no apodization or grating chirp

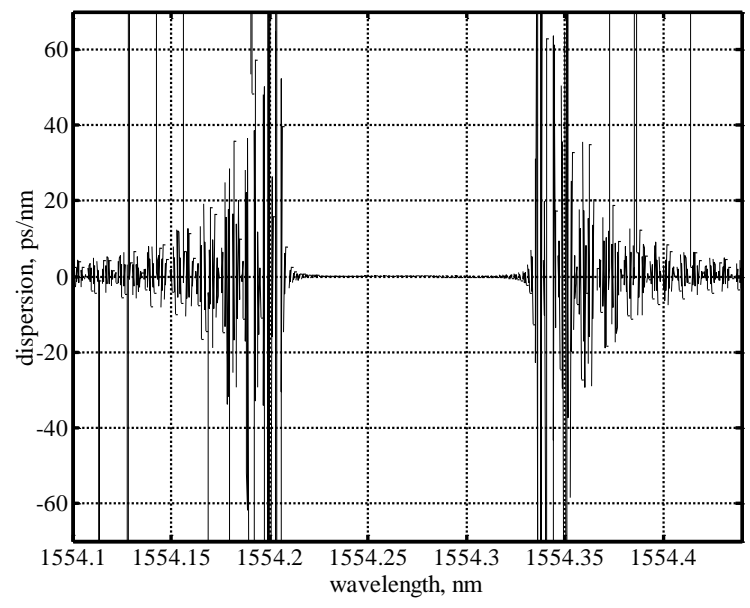

Figure 8. Dispersion in the area of the wavelength of the compensator's Bragg grating with no apodization or grating chirp

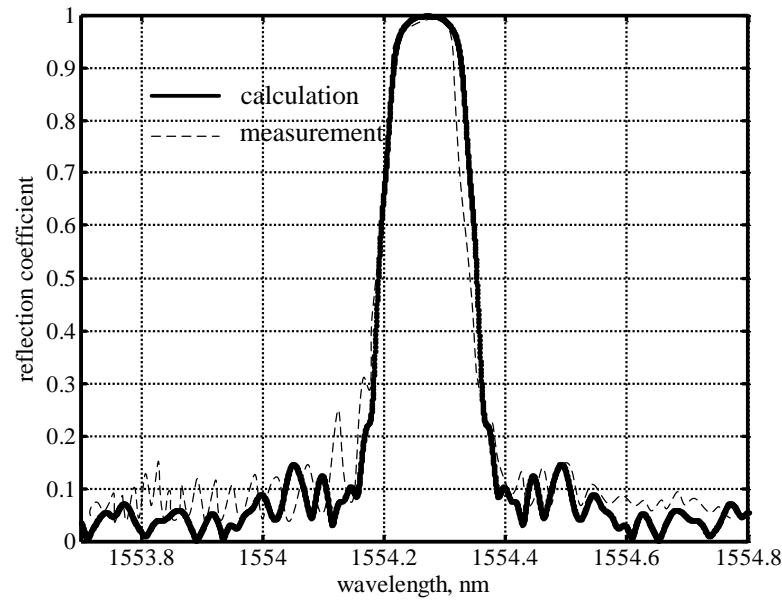

Figure 9. Measured and calculated transmission spectrum with no grating chirp when the Gaussian apodization function $(a=10)$ is applied

(a)

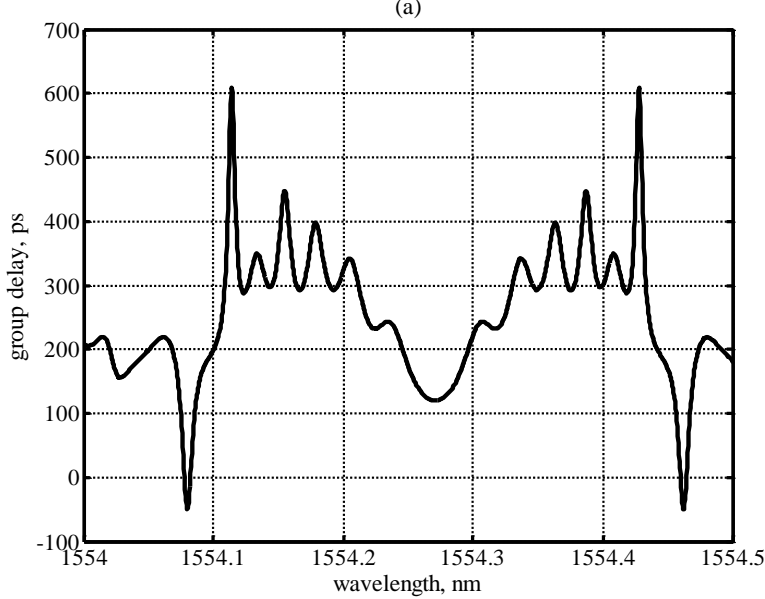


(b)

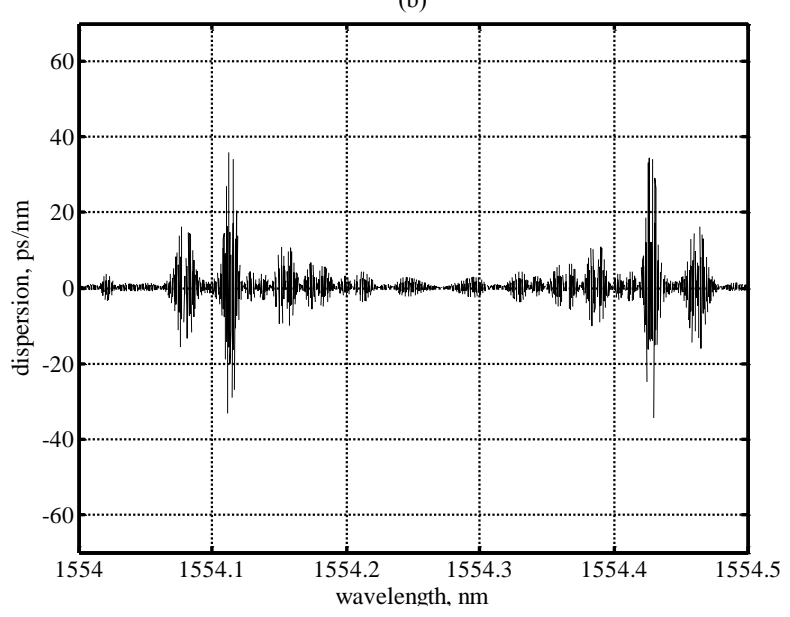

Figure 10. Characteristics obtained without grating chirp when the Gaussian apodization function $(a=10)$ is applied.

(a) group delay, (b) dispersion

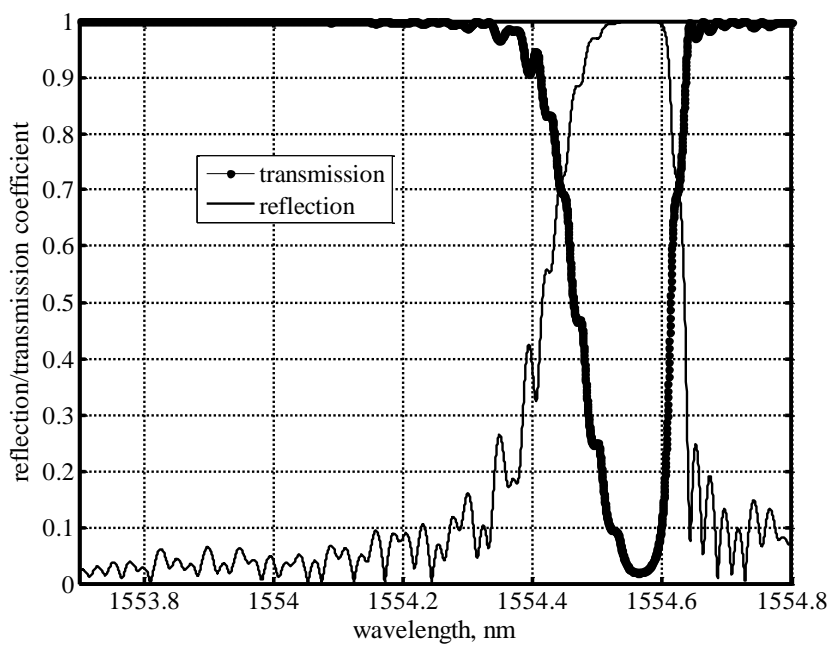

Figure 11. Transmission and reflection spectrum in the case of heterogeneous elongation of the compensator's grating. The maximum value of the grating's deformation $=1.95 \cdot 10^{-5} \varepsilon$. The grating is apodized according tothe Gaussian function $(a=5)$

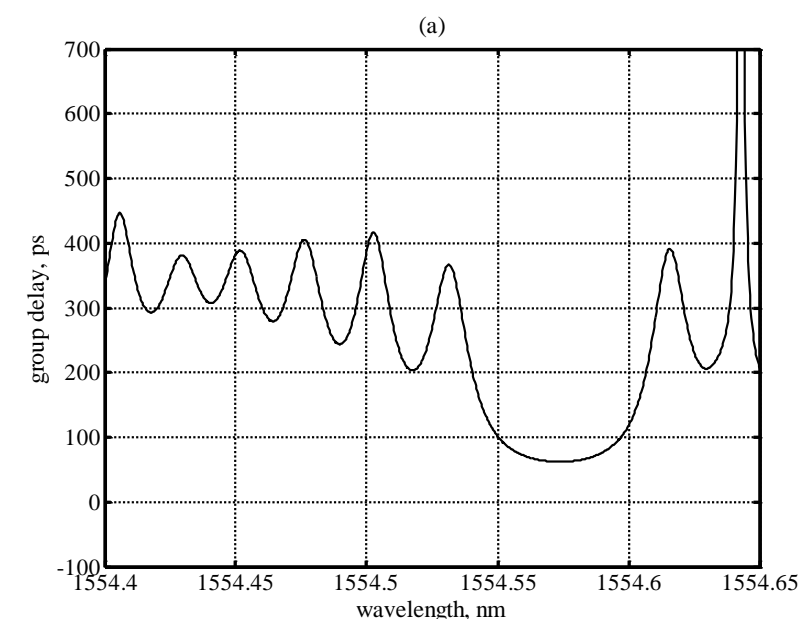

(b)

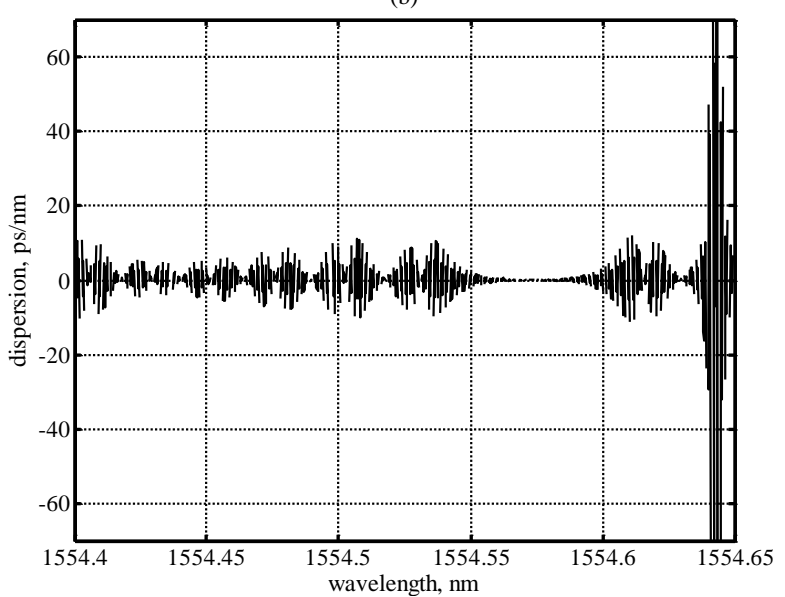

Figure 12. Characteristics obtained in the case of heterogeneous elongation of the compensator's grating. The maximum value of the grating's deformation $=1.95 \cdot 10^{-5} \varepsilon$. The grating is apodized according to the Gaussian function $(a=5)$, (a) group delay, (b) dispersion

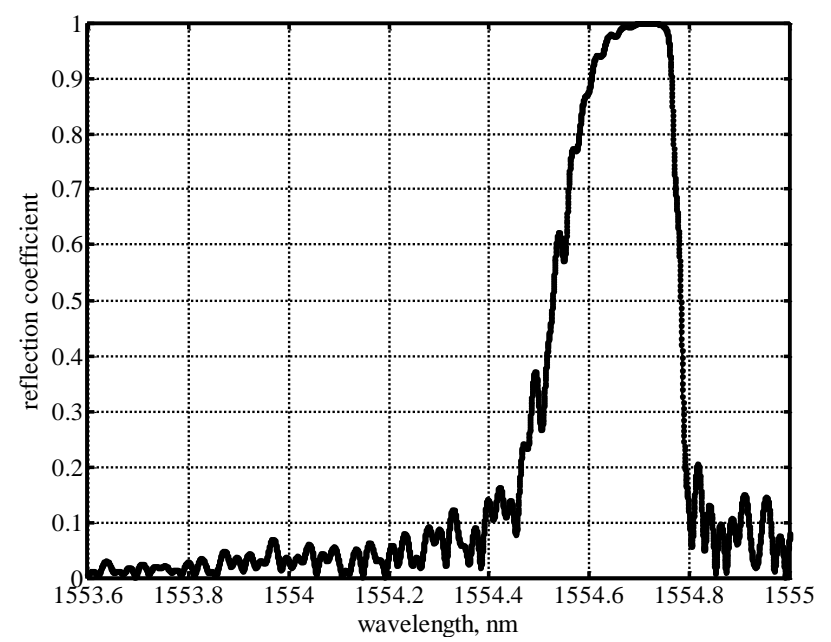

Figure 13. Reflection spectrum in the case of heterogeneous elongation of the compensator's grating. The maximum value of the grating's deformation $=$ $2.92 \cdot 10^{-5} \varepsilon$. The grating is apodized according to the Gaussian function $(a=5)$

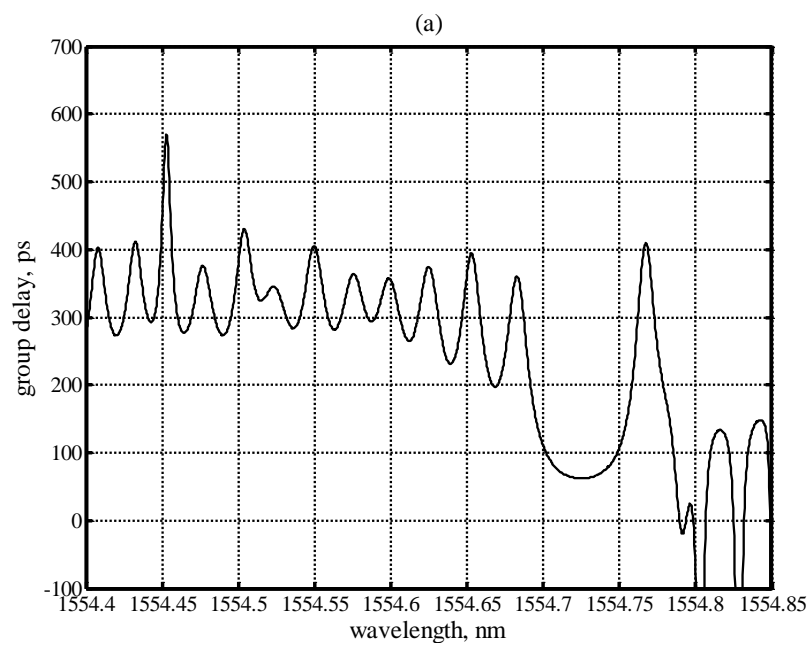




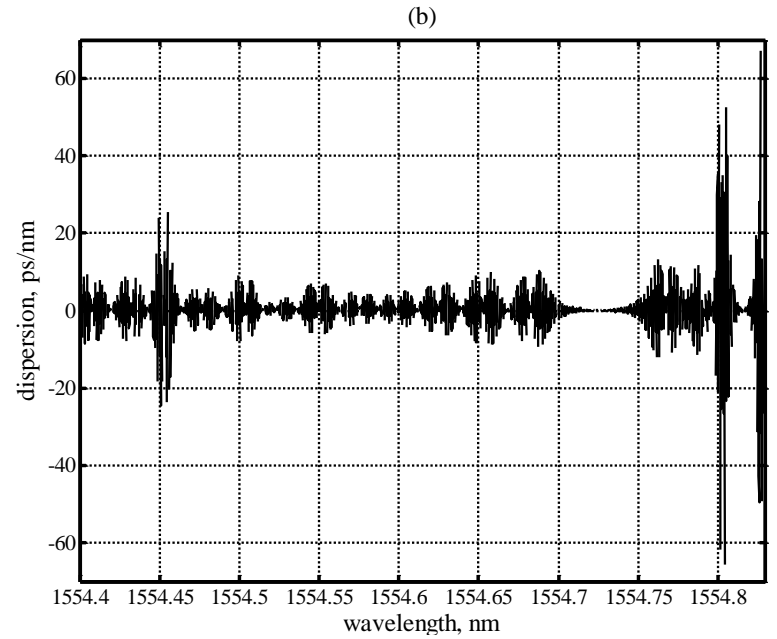

Figure 14. Reflection spectrum in the case of heterogeneous elongation of the compensator's grating. The maximum value of the grating's deformation $=2.92 \cdot 10^{-}$

${ }^{5} \varepsilon$. The grating is apodized according to the Gaussian function ( $a=5$ ), (a) group delay, (b) dispersion

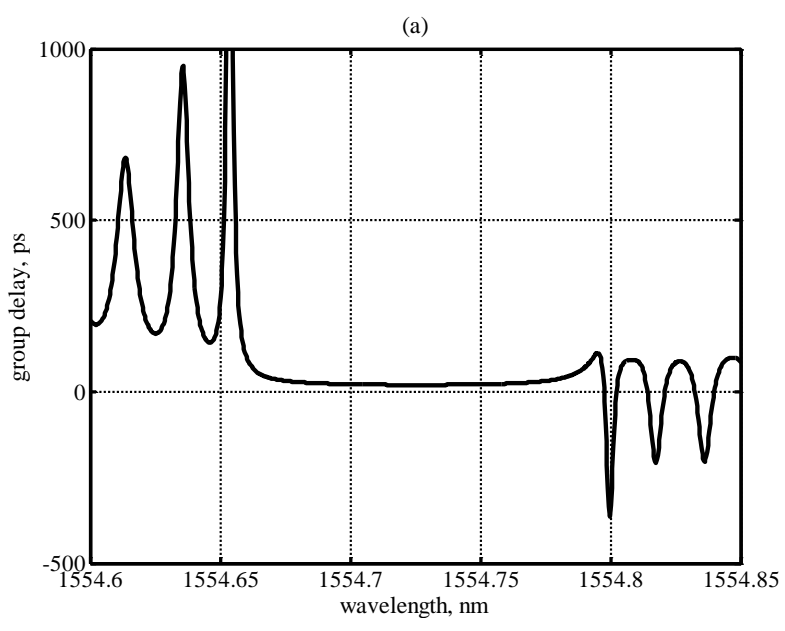

(c)

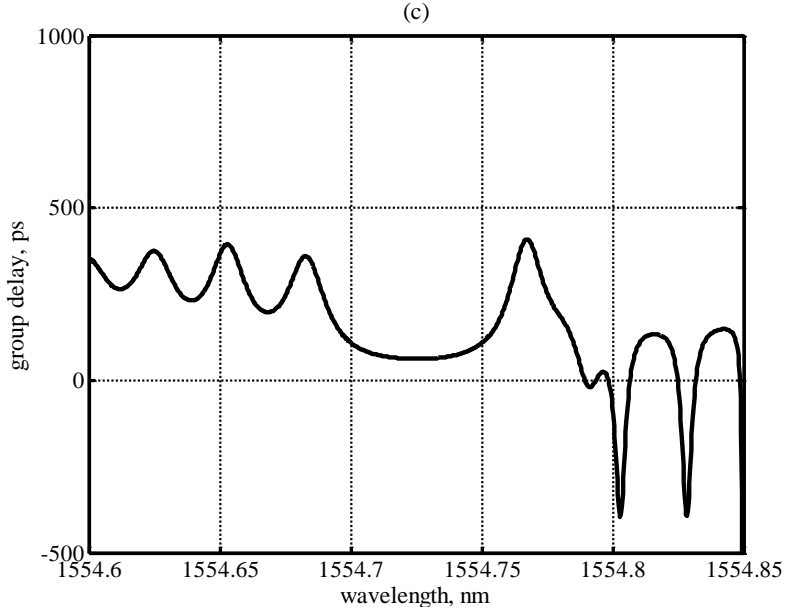

(b)

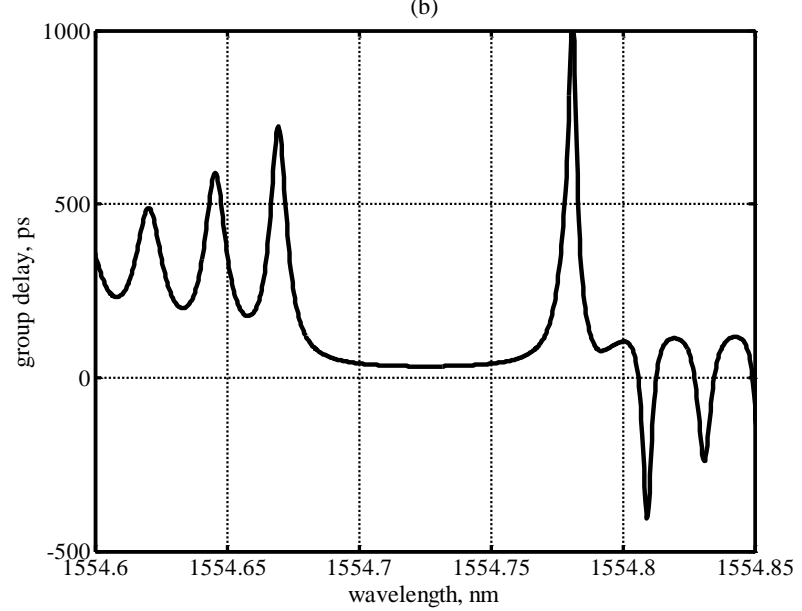

(d)

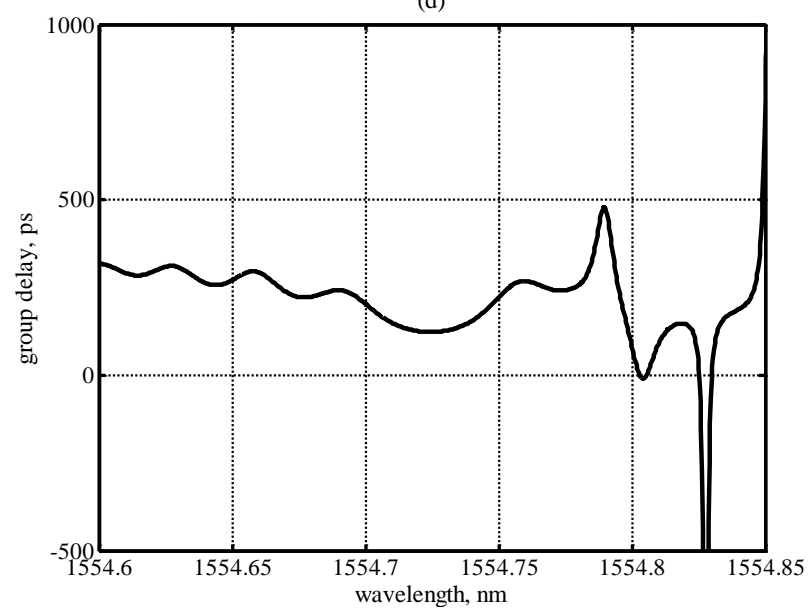

Figure 15. Graphs of group delay in the vicinity of the dispersion compensator's Bragg wavelength,

(a) no apodization, (b) Gauss apodization, $a=2$, (c) Gauss apodization, $a=5$, (b) Gauss apodization, $a=10$,

The illustrations above show the relationship between group delay and dispersion for various values of the maximum value of the heterogeneous deformation to which the proposed dispersion compensator system was subjected. An analysis was made of the relationship of the dispersion characteristics depending on the apodization profile of the
Bragg grating. The spectral characteristics of the system's transmission and reflection were compiled in order to demonstrate the change and shift in the spectrum width, which also affected the system's dispersion properties. As can be seen, the increase in amplitude of the deformation causes a broadening of the reflective and transmission 
spectral characteristics of the compensator. On the basis of the characteristics displayed in figures 8, 10, 12 and 14 a clear decrease can be seen in the dispersion values in the range of the Bragg wavelengths. The fields in which the lowest dispersion values appear coincide with the lengths of waves which meet the Bragg condition, which are in turn illustrated in figures $6,9,11$ and 13 .

The results collated in figure 18 demonstrated that it is possible to control the gradient of the group delay curve by simply regulating parameter $a$ of the given Gauss distribution by relationship (20).It can be seen that along with the increase in the parameter from 0 to 10 , this band is reduced and the gradient of the group delay curve decreases, causing a weakening of the dispersion compensation effect. The dynamics of the change in group delay for the nonapodized grating measures 500 ps, while in the case of Gaussian apodization with the parameter $a=10$ this decreases to $\sim 300 \mathrm{ps}$. The characteristics from figure 15 also show that along with the increase in parameter $a$ of the grating's apodization function, the group delay characteristic is smoothed out.

\section{CONCLUSIONS}

A dispersion compensator was presented which is tuned by the heterogeneous stress of a Bragg grating. Analysis and simulation tests demonstrated that the level of the maximum value of the heterogeneous stress of the grating affects the group delay characteristics and dispersion. It can also be noted that the use of apodization affects the size of the area where the group delay characteristic is flat. By using the negative gradient properties of the group delay characteristic of the proposed system it is possible to apply it in accumulated dispersion compensation in fast data transmission systems.

The additionally presented results prove that along with an increase in the maximum value of the heterogeneous deformation of the grating there is an increase in the scope of the wavelengths for which the value of the group delay is a falling curve, there is a simultaneous shift in the wavelengths for which a significant limiting of dispersion takes place. This change is caused by a shifting of the wavelength of the Bragg grating, which is subjected to mechanical stretching. In cases where a Bragg grating is used as a dispersion compensator, the influence of the temperature is highly significant, so developing a method for controlling the temperature or eliminating its influence would seem to be of major importance.

\section{REFERENCES}

[1] R. Tewari, M. Basu, H. N. Acharya, "Studying the effect of a central dip on the performance of a dispersion compensated fibre," Optics Communications vol. 174, pp. 405-411, 2000

[2] M. Jablonski, Y. Takushima, K. Kikuchi, Y. Tanaka, K. Furuki, K. Sato, N. Higashi, „Layered optical thin-film allpass dispersion equaliser for compensation of dispersion slope of optical fibres," Electronic Letters vol. 36(13), pp. 1139-1141, 2000
[3] Z. Gotra, O. Gotra, Z. Mikityuk, R. Zayats, B. Stadnyc, "Fibre-optic sensors on the base of liquid crystals," Lightguides and Their Applications, proceedings of SPIE 4239:76-81, 2000

[4] Z. Mikityuk, I. Lopatynskiy, O. Gotra, B. Dalanbalar, S.V. Svenchnikov. V. P. Kojemiako, S.A. Kostyukevych, "Liquid crystal using in optical sensors," Selected papers from international conference on optoelectronic information technologies, proceedings of SPIE 4425:472-477, 2001

[5] M. Akbulut, A. M. Weiner, P. J. Miller, "Broadband All-Order Polarization Mode Dispersion Compensation Using Liquid-Crystal Modulator Arrays," Journal of Lightwave Technology vol. 24(1), pp. $251-, 2006$

[6] Y. Yin, X. Chen, L. Zhu, K. Xu, Z. Lin, X. Li, S. Xie, "Analytical expression and system simulation of eye opening penalty for chirped sampled Bragg grating dis[ersion compensator," Optics Communications vol. 203, pp. 93-99, 2002

[7] N. Q. Ngo, S. Y. Li, R. T. Zheng, S. C. Tjin, P. Shum, "Electrically Tunable Dispersion Compensator With Fixed Center Wavelength Using Fiber Bragg Grating," Journal of Lightwave Technology vol. 21(6), pp. 1568-1575, 2003

[8] Z. Tan, C. Yong, Y. Liu, T. Zhi, C. Jihong, N. Tigang, et al. "Crossphase modulation in long-haul systems with chirped fiber Bragg gratings-based dispersion compensators," Optik vol. 118, pp. 216-220, 2007

[9] G. Ning, S.Aditya, P. Shum, Y.D. Gong, X.Y. Dong, J.H. Ng, "A PMD compensator with Hi-Bi chirped FBG free from chromatic dispersion," Optics Communications vol. 245, pp. 153-157, 2005

[10] Y. J. Lee, J. Bae, K. Lee, J. M. Jeong, S. B. Lee, “Tunable Dispersion and Dispersion Slope Compensator Using Strain-Chirped Fiber Bragg Grating,” IEEE Photonics Technology Letters vol. 19(10), pp. 762-764, 2007

[11] S. S. A. Khan, M. S. Islam, "Performance evaluation of different apodization profiles of linearly chirped FBG for dispersion compensation," Computer and Information Technology (ICCIT), pp. 350-354, 2011

[12] M. F. Ferreira, A. N. Pinto, P. S. Andre, N. J. Muga, J. E. Machaldo, R. N. Nogueira, et al. "Polarization Mode Dispersion in High-Speed Optical Communication Systems," Fiber and Integrated Optics vol. 24(3-4), pp. 261-285, 2005

[13] S. A. Kolpakov, Yu. O. Barmenkov, V. Aboites, "Asymmetrically Apodized Fiber Bragg Gratings for Applications in Dispersion-Less Fabry-Perot Fiber Cavities," Fiber and Integrated Optics vol. 29(6), pp. 466-479, 2010

[14] M. Li, H. Li, "Reflection equalization of the simultaneous dispersion and dispersion-slope compensator based on a phase-only sampled fiber Bragg grating, “ Optics Express vol. 16(13), pp. 9821-9828, 2008

[15] F. Karim, O. Seddiki, "Study on raised-cosine sampled chirped fiber Bragg grating for dispersion compensation applications," Computer and Simulation in Modern Science, pp. 97-99, 2009

[16] M. J. Erro, M. A. G. Laso, D. Benito, E. M. J. Gard, M. A. Muriel, "Third-Order Dispersion in Linearly Chirped Bragg Gratings and Its Compensation," Fiber and Integrated Optics vol. 19(4), pp. 367-382, 2000

[17] C. A. F. Marques, R. A. Oliveira, J. Canning, A. A. P. Pohl, R. N. Nogueira, "Controlling the properties of Fiber Bragg Gratings based on the Acousto-Optic modulation," EUROCON - International Conference on Computer as a Tool, IEEE 1-3, 2011

[18] P. Kisała, "Metrological conditions of strain measurement optoelectronic method by the use of fibre Bragg gratings," Metrology and Measurement Systems vol. 19(3), pp. 471-480, 2012

[19] .P. Kisała, "Generation of a zone chirp in uniform Bragg grating as a way of obtaining double functionality of a sensor," Metrology and Measurement Systems vol. 19(4), pp. 727-738, 2012

[20] P. Kisała, S. Cieszczyk, "Method of simultaneous measurement of two direction force and temperature using FBG sensor head," Applied Optics vol. 54(10), pp. 2677-2687, 2015

[21] R. Kashyap, "Fiber Bragg Gratings," Academic Press, New York, 19 